\title{
RNA polymerase II CTD phospho-sites Ser5 and Ser7 govern phosphate homeostasis in fission yeast
}

\author{
BEATE SCHWER, ${ }^{1}$ ANA M. SANCHEZ, ${ }^{1}$ and STEWART SHUMAN ${ }^{2}$ \\ ${ }^{1}$ Department of Microbiology and Immunology, Weill Cornell Medical College, New York, New York 10065, USA \\ ${ }^{2}$ Molecular Biology Program, Sloan-Kettering Institute, New York, New York 10065, USA
}

\begin{abstract}
Phosphorylation of the tandem YSPTSPS repeats of the RNA polymerase II CTD inscribes an informational code that orchestrates eukaryal mRNA synthesis. Here we interrogate the role of the CTD in phosphate homeostasis in fission yeast. Expression of Pho1 acid phosphatase, which is repressed during growth in phosphate-rich medium and induced by phosphate starvation, is governed strongly by CTD phosphorylation status, but not by CTD repeat length. Inability to place a Ser7- $\mathrm{PO}_{4}$ mark (as in $\left.S 7 A\right)$ results in constitutive derepression of $\mathrm{Pho} 1$ expression in phosphate-replete medium. In contrast, indelible installation of a Ser7- $\mathrm{PO}_{4}$ mimetic (as in $S 7 E$ ) hyper-represses Pho1 in phosphate-replete cells and inhibits Pho1 induction during starvation. Pho1 phosphatase is derepressed by ablation of the CTD Ser5-PO mark, achieved either by mutating Ser5 in all consensus heptads to alanine, or replacing all Pro6 residues with alanine. We find that Ser5 status is a tunable determinant of Pho1 regulation, i.e., serial decrements in the number of consensus Ser5 heptads from seven to two elicits a progressive increase in Pho1 expression in phosphate-replete medium. Pho1 is also derepressed by hypomorphic mutations of the CTD kinase Cdk9. Inactivation of the CTD phosphatase Ssu72 attenuates Pho1 induction in wild-type cells and blocks Pho1 derepression in S7A cells. These experiments implicate Ser5, Pro6, and Ser7 as component letters of a CTD coding "word" that transduces a repressive transcriptional signal via serine phosphorylation.
\end{abstract}

Keywords: phosphate-responsive gene expression; acid phosphatase; phosphate transporter

\section{INTRODUCTION}

Phosphate homeostasis in the fission yeast Schizosaccharomyces pombe is governed by an intricate signaling network entailing positive and negative influences on the transcription of genes encoding proteins involved in extracellular phosphate mobilization and uptake, specifically a cell surface acid phosphatase Phol and an inorganic phosphate transporter Pho84 (Carter-O'Connell et al. 2012). Upregulation of the expression of these genes during phosphate starvation depends on the transcription factor Pho7 (Henry et al. 2011; Carter-O'Connell et al. 2012), a member of the zinc binuclear cluster family of fungal DNA-binding transcription regulators (MacPherson et al. 2006). The protein kinase Csk1 is a negative regulator of the phosphate response, as judged by the fact that phol expression is constitutively turned on in csk $1 \Delta$ cells under phosphate-replete conditions (Henry et al. 2011; Carter-O'Connell et al. 2012; Schwer et al. 2014). Csk1 is a CDK-activating kinase with several physiological targets, including the kinases Cdc2, Mcs6, and Cdk9 (Saiz and Fisher 2002; Pei et al. 2006). Mcs6 and Cdk9 figure

Corresponding authors: s-shuman@ski.mskcc.org, bschwer@med. cornell.edu

Article published online ahead of print. Article and publication date are at http://www.rnajournal.org/cgi/doi/10.1261/rna.052555.115. prominently in fission yeast transcription via their phosphorylation of the carboxy-terminal domain (CTD) of the Rpb1 subunit of RNA polymerase II (Pol2) and/or the carboxy-terminal domain (CTD) of the transcription elongation factor Spt5 (Pei et al. 2006; Viladevall et al. 2009).

The Pol2 CTD consists of tandemly repeated heptapeptides of consensus sequence $\mathrm{Y}^{1} \mathrm{~S}^{2} \mathrm{P}^{3} \mathrm{~T}^{4} \mathrm{~S}^{5} \mathrm{P}^{6} \mathrm{~S}^{7}$. The inherently plastic CTD structure is modulated by phosphorylation of the Tyr1, Ser2, Thr4, Ser5, and Ser7 residues and by cis-trans isomerization of the prolines (Corden 2013; Eick and Geyer 2013). With up to $128^{n}$ potential CTD primary structures (where $n$ is the number of heptads), the CTD provides information about the state of the transcription machinery-a CTD code-that is "read" by diverse CTD receptor proteins that control transcription, modify chromatin structure, and catalyze or regulate mRNA capping, splicing, and polyadenylation (Corden 2013; Eick and Geyer 2013).

Informational rules for the CTD code in fission yeast have been elucidated by genetically manipulating the composition

(C) 2015 Schwer et al. This article is distributed exclusively by the RNA Society for the first 12 months after the full-issue publication date (see http://rnajournal.cshlp.org/site/misc/terms.xhtml). After 12 months, it is available under a Creative Commons License (Attribution-NonCommercial 4.0 International), as described at http://creativecommons.org/licenses/ by-nc/4.0/. 
and structure of the Pol2 CTD, which consists of 25 YSPTSPS repeats connected to the body of the Rpb1 subunit via four degenerate repeats (the CTD "rump") that deviate in size and/or sequence from the consensus heptad. A CTD composed of the rump plus 12 or more native heptads suffices for normal growth of Schizosaccharomyces pombe under all laboratory conditions tested (Schneider et al. 2010). By introducing alanines and conservative mutations in lieu of Tyr 1 , Ser2, Pro3, Thr4, Ser5, Pro6, and Ser7 in every consensus heptad of a fully functional Rpb1-CTD array (comprising the rump plus 14 consensus heptad repeats), we determined that (i) Tyr1, Pro3, Ser5, and Pro6 are essential for viability, by the criterion that alanine substitution is lethal, whereas Ser2, Thr4, and Ser7 are not essential; and (ii) $Y 1 F, Y 1 F+$ $S 7 A, S 2 A+S 7 A$, and $T 4 A+S 7 A$ mutants are viable, signifying that phenylalanine is functional in lieu of Tyr1 and that Ser5 is the only strictly essential phosphorylation site in fission yeast (Schwer and Shuman 2011; Schwer et al. 2012).

A connection between the Pol2 CTD and phosphate homeostasis emerged when we applied RNA-seq to gauge globally the impact of the loss of the four inessential CTD phosphoacceptors. This analysis illuminated how individual letters of the Pol2 CTD code affect the expression of limited and distinct sets of genes. To wit, CTD mutations $S 2 A, Y 1 F$, $S 7 A$, and $T 4 A$ elicited $\geq$ twofold dysregulation of only $4.4 \%$, $1.4 \%, 1.2 \%$, and $0.14 \%$ of the annotated fission yeast protein-coding RNAs, respectively (Schwer et al. 2014). CTD letters Thr4 and Ser7 were identified as novel components of the phosphate response pathway, on which the T4A and $S 7 A$ mutations had opposing effects. T4A reduced the expression of the genes encoding Phol and the Pho84 (without affecting the expression of the pho7 or cskl genes that regulate the phosphate response pathway), while S7A increased Phol expression (Schwer et al. 2014).

Prompted by these initial findings, we aim here to further interrogate the role of the Pol2 CTD in the expression of Phol acid phosphatase during phosphate-replete and phosphate-starved states and in the transcriptional control of the phosphate-responsive phol and pho84 genes. We address the effects of (i) CTD length; (ii) single and combinatorial subtraction of potential phosphorylation sites, including Ser5; (iii) genetic manipulation of kinases; and (iv) inactivation of the CTD phosphatase Ssu72. Our results implicate Ser5, Pro6, and Ser7 as letters in a coding "word" that specifies repression of the phosphate regulon when phosphate is replete.

\section{RESULTS}

\section{Effect of Pol2 CTD length on regulated Pho1 expression}

To determine the impact of the CTD length on the induction of Phol under conditions of phosphate starvation, we exploited a set of fission yeast strains with chromosomal $r p b 1$ alleles encoding either the native wild-type CTD array (29 re- peats, consisting of the rump plus 25 consensus heptads) or CTDs serially truncated at their $\mathrm{C}$ termini so as to comprise $26,20,18,16$, or 13 repeats (i.e., the rump plus $22,16,14,12$, or 9 consensus heptads) (Schneider et al. 2010). Cells grown in liquid culture in YES medium were washed and then incubated for $3 \mathrm{~h}$ either in synthetic medium containing $15.5 \mathrm{mM}$ phosphate (phosphate-replete) or in medium lacking exogenous phosphate to elicit the starvation response. Acid phosphatase activity (a gauge of Phol enzyme level) was quantified by incubating suspensions of serial dilutions of the phosphate-replete or phosphate-starved cells for $5 \mathrm{~min}$ with $p$-nitrophenylphosphate and assaying colorimetrically the formation of $p$-nitrophenol. The basal phosphatase activity of wild-type $r p b 1$ cells with 29 repeats was increased 4.5 -fold by phosphate starvation (Supplemental Fig. S1). Truncating the CTD had little effect on Phol activity under phosphate-replete conditions (Supplemental Fig. S1). CTD truncations also had little effect on the induction of Phol activity by phosphate starvation (Supplemental Fig. S1). Thus, CTD length is not a key determinant of the phosphate starvation response.

\section{Effect of eliminating CTD phosphorylation sites, individually and pairwise}

We surveyed our ensemble of viable rpb1-CTD mutants for effects on the Phol phosphate starvation response. The "wild-type" CTD control in these experiments consists of the rump plus 14 consensus heptads; the mutants have alanine or a conservative substitution in every one of the 14 heptads, either singly or in pairwise combinations (Schwer and Shuman 2011; Schwer et al. 2012). Phosphatase activity of rpb1-CTD-WT cells was increased fivefold by phosphate starvation (Fig. 1). Eliminating the Tyr1 or Ser2 hydroxyl groups

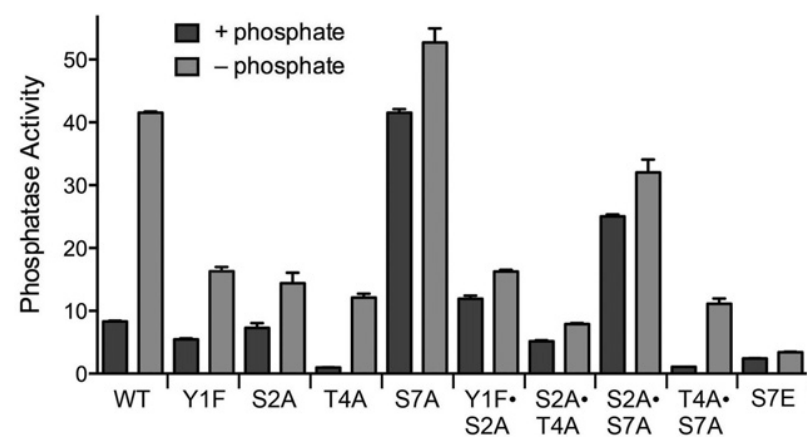

FIGURE 1. Effect of eliminating CTD phosphorylation sites on phosphate homeostasis. Isogenic heterothallic S. pombe rpb1-CTD strains in which 14 heptad repeats (either wild-type YSPTSPS or mutated heptads as specified) are appended to amino acid 1577 of $\operatorname{Rpb} 1(11,12)$ were grown in liquid culture in YES medium. Cells were then harvested, washed with water, and incubated for $3 \mathrm{~h}$ in PMG medium containing $15.5 \mathrm{mM}$ phosphate (+ phosphate) or lacking exogenous phosphate (- phosphate). Phol phosphatase was assayed by conversion of $p$-nitrophenylphosphate to $p$-nitrophenol. The $y$-axis specifies the phosphatase activity $\left(A_{410}\right)$ normalized to input cells $\left(A_{600}\right)$. 
in the Y1F and S2A strains had little effect on phosphatase activity under phosphate-replete conditions, but it did dampen the induction of Phol after $3 \mathrm{~h}$ of phosphate depletion, to $39 \%$ and $35 \%$ of the wild-type control (Fig. 1). When we tested a Y1F•S2A double mutant, we found that basal phosphatase activity was increased slightly, but the phosphatase activity after phosphate depletion remained at the same diminished level seen in the $Y 1 F$ and $S 2 A$ single mutants (Fig. 1). Thus, Tyr1 and Ser2 act concordantly to promote the induction of Phol during phosphate starvation.

The basal phosphatase activity of T4A cells in phosphaterich medium was eightfold lower than wild type. T4A cells responded to phosphate starvation (12-fold induction), but the level of induced phosphatase activity was only $29 \%$ that of wild-type cells $3 \mathrm{~h}$ post-starvation. Thus, the Thr4 CTD mark exerts a positive effect on Phol expression, independent of exogenous phosphate status. In an $S 2 A \cdot T 4 A$ double mutant, basal phosphatase activity was largely restored, but there was hardly any increase in Phol activity at $3 \mathrm{~h}$ after phosphate depletion (Fig. 1).

In contrast, $S 7 A$ cells displayed a high level of phosphatase activity in phosphate-rich medium, equivalent to the Phol activity of wild-type cells that were phosphate-starved for $3 \mathrm{~h}$ (Fig. 1), signifying that Ser7 is essential to repress Pho1 expression when phosphate is available. $S 7 A$ cells further increased their phosphatase activity by $25 \%$ at $3 \mathrm{~h}$ after phosphate depletion. As noted previously (Schwer et al. 2014), the super-induction of Phol by S7A was eliminated in the $T 4 A \cdot S 7 A$ strain, in which the low basal and induced phosphatase activities echoed the T4A single mutant (Fig. 1). Apparently, the Thr4 mark is important for Phol expression even when a repressive signal is removed. In the $S 2 A \cdot S 7 A$ double mutant, we found that the derepressed pattern characteristic of $S 7 A$ was maintained, albeit at lower magnitude, i.e., the basal phosphatase activity in $S 2 A \cdot S 7 A$ cells was threefold higher than wild-type, but $60 \%$ less than S7A (Fig. 1).

An instructive finding emerged from analysis of the $r p b 1$ CTD-S7E strain, in which Ser7 in all heptads was replaced by the phosphomimetic glutamic acid. S7E cells had diminished basal phosphatase activity and failed to mount an inductive response after $3 \mathrm{~h}$ of phosphate starvation (Fig. 1). It would appear that Phol expression is governed strongly by CTD Ser7 status, whereby inability to place a Ser7 $-\mathrm{PO}_{4}$ mark (as in S7A) results in constitutive derepression of Phol, and indelible installation of a Ser7- $\mathrm{PO}_{4}$ mimetic (as in S7E) inhibits Phol induction.

\section{Contribution of CTD Ser5 to regulated Pho1 expression}

In light of the findings above regarding the importance of Ser7 in Phol regula- tion, we aimed to gauge the impact of altered Ser5 content on phosphate homeostasis. Ser5 is the only phosphoacceptor amino acid in the Pol2 CTD that is strictly essential for growth of fission yeast. Replacing all of the Ser5 residues in the consensus heptads with alanine is lethal (Schwer and Shuman 2011). Through the analysis of chimeric CTDs, composed of the rump plus a total of 14 Ser5-containing and Ala5-containing heptads, in assorted combinations, we showed that as few as two Ser5 heptads sufficed for vegetative growth (Schwer et al. 2012). Immunoblotting cellular Rpb1 with phospho-specific antibodies showed that the intensity of the Ser5- $\mathrm{PO}_{4}$ mark was proportional to the number of Ser5 heptads in the CTD. Furthermore, the intensity of the Ser7 $-\mathrm{PO}_{4}$ mark also correlated with the number of Ser5 heptads (Schwer et al. 2012). Therefore, we asked whether reduced Ser5 content might elicit a derepression of Pho1 akin to what we observed in S7A cells.

At the outset, we compared Phol regulation in wild-type rpb1-CTD-(S5) $)_{14}$ cells to that of three different chimeras in which the CTD contained a mixture of seven S5 heptads and seven S5A heptads, arrayed such that (i) the S5 and S5A heptads alternated (with heptads 1, 3, 5, etc. being S5A and 2, 4, 6, etc. being S5), (ii) the proximal half were S5A and the distal half were S5, or (iii) the proximal 7 heptads were S5 and the distal 7 heptads were S5A. The $(S 5 A \cdot S 5)_{7}$, $(\mathrm{S} 5 \mathrm{~A})_{7}(\mathrm{~S} 5)_{7}$, and $(\mathrm{S} 5)_{7}(\mathrm{~S} 5 \mathrm{~A})_{7}$ strains displayed seven-, six-, and fourfold greater phosphatase activity than wild-type $(S 5)_{14}$ cells under phosphate-replete conditions and they elaborated greater than wild-type phosphatase activity in response to $3 \mathrm{~h}$ of phosphate starvation (Fig. 2A). Thus, even a $50 \%$ reduction in the Ser5 content of the 14-heptad CTD perturbed the phosphate regulatory axis.

We then proceeded to survey a series of chimeric CTDs consisting of $6,5,4,3$, or 2 Ser5-containing heptads linked to $8,9,10,11$, or $12 \mathrm{~S} 5 \mathrm{~A}$ heptads, respectively. The salient findings were that serial decrements in the number of consensus Ser5 heptads from seven to two elicited a progressive increase in Phol expression in phosphate-replete medium
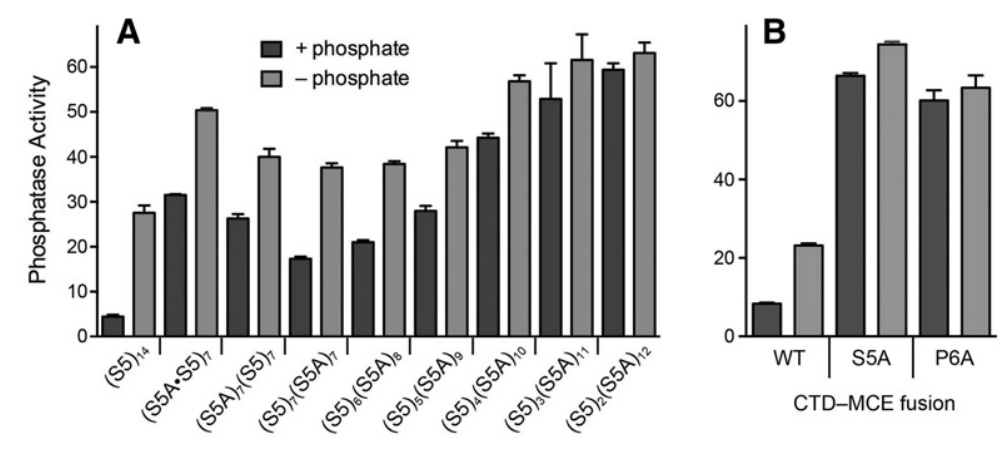

FIGURE 2. Contribution of CTD Ser5 to regulated Pho1 expression. Cells with Rpb1-CTDs composed of the indicated mixtures of S5 heptads and S5A heptads (A), or CTD-MCE fusions in which the 14 heptads were all wild-type, S5A, or P6A $(B)$, were assayed for Phol activity after incubation for $3 \mathrm{~h}$ in PMG medium containing $15.5 \mathrm{mM}$ phosphate (+ phosphate) or lacking exogenous phosphate (- phosphate). 
(Fig. 2A). Indeed, the basal activity of $(S 5)_{2}(S 5 A)_{12}$ cells was 13 -fold greater than the wild-type $(S 5)_{14}$ cells. The level of phosphatase activity in $(S 5)_{2}(S 5 A)_{12}$ cells did not increase after $3 \mathrm{~h}$ of phosphate starvation (Fig. 2A).

Replacing all of the Ser5 residues in the consensus heptads with alanine is lethal because the Ser5- $\mathrm{PO}_{4}$ mark is needed for recruitment of the fission yeast mRNA capping enzymes RNA triphosphatase and RNA guanylyltransferase to the Pol2 elongation complex (Pei et al. 2003; Schwer and Shuman 2011; Doamekpor et al. 2014). Replacing all of the Pro6 residues in the consensus heptads with alanine is lethal because Pro6 is necessary to inscribe the Ser5$\mathrm{PO}_{4}$ mark needed for capping enzyme recruitment (Schwer et al. 2012). The lethality of $r p b 1-C T D-S 5 A$ and $r p b 1-C T D-$ $P 6 A$ can be rescued by covalently fusing mammalian capping enzyme (MCE, a bifunctional RNA triphosphatase-guanylyltransferase) to the mutant Rpb1S5A and Rpb1-P6A polypeptides. We find here that Phol expression in the rpb1-CTD-S5A-MCE and rpb1-CTD$P 6 A-M C E$ fusion strains was derepressed in phosphate-replete medium vis-à-vis the control rpb1-CTD-WT-MCE fusion strain (Fig. 2B).

These experiments establish that CTD coding letters Ser5, Pro6, and Ser7 are essential for the repression of Phol activity under phosphate-rich conditions.

\section{Mapping the $5^{\prime}$ ends of mRNAs and a noncoding RNA transcribed from a clustered phosphate response regulon}

The fission yeast phosphate transporter Pho84 and the acid phosphatase Phol are encoded by adjacent co-oriented genes on chromosome II (Fig. 3A). Previously, we used reverse transcriptase primer extension analysis to map the $5^{\prime}$ end of the pho1 mRNA to a single start site 51 nucleotides upstream of the AUG start codon of the pho1 open reading frame (Schwer et al. 2014). Here, we used the same strategy to locate the $5^{\prime}$ end of the neighboring phos4 gene. A ${ }^{32} \mathrm{P}$-labeled DNA primer complementary to nucleotides -87 to -108 upstream of the phos4 open reading frame was annealed to total yeast RNA and then subjected to reverse transcription. The RT primer extension product was analyzed by denaturing PAGE in parallel with a by autoradiography. chain-terminated sequencing ladder generated by DNA polymerase-catalyzed extension of the same ${ }^{32} \mathrm{P}$-labeled primer annealed to a DNA template extending from nucleotides -500 to +130 of the transporter gene. Two $5^{\prime}$ ends were thereby located 151 and 149 nt upstream of the start codon of the pho84 open reading frame (Fig. 3B).

Two recent studies have identified in fission yeast $r r p 6 \Delta$ cells (which lack a catalytic subunit of the nuclear exosome) a long noncoding (lnc) RNA transcribed from the $\sim 1.6-\mathrm{kb}$
A

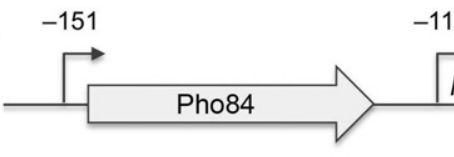

\section{B}
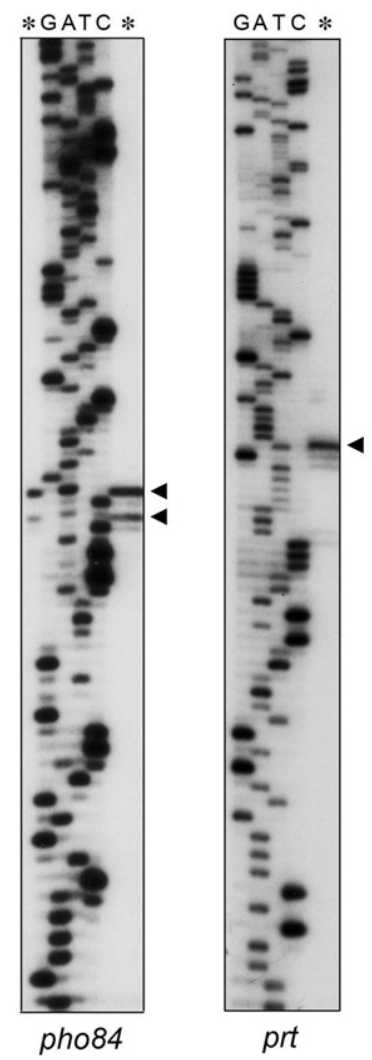

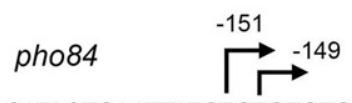

CATACTGAATTATCTGTGTGTGTAAACCACC
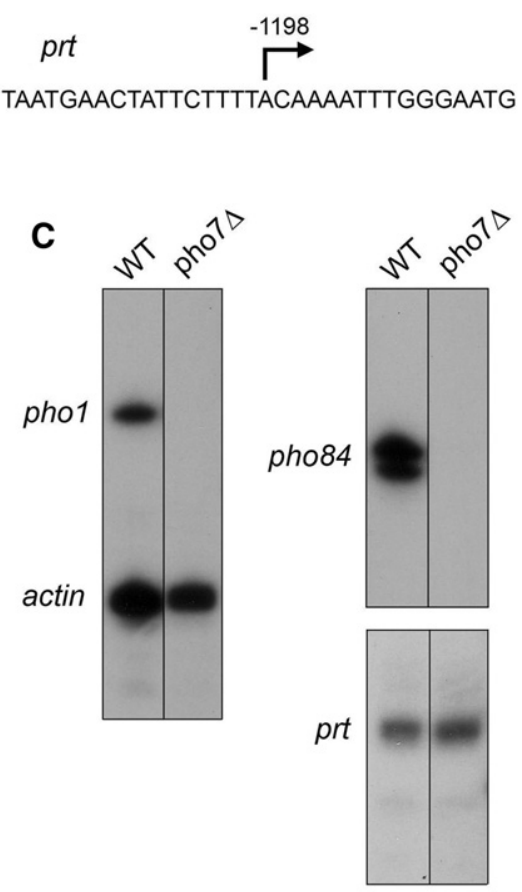

FIGURE 3. 5 -end mapping of RNAs transcribed from the fission yeast phosphate regulon. $(A)$ Schematic illustration of the adjacent $p h o 84^{+}(\mathrm{SPBC} 8 \mathrm{E} 4.01 \mathrm{c})$ and $p h o 1^{+}$(SPBP4G3.02) genes on fission yeast chromosome II, with the open reading frames denoted by arrows in the direction of mRNA synthesis. The experimentally mapped $5^{\prime}$ ends of the pho84 and pho1 mRNAs and the intervening noncoding prt RNA are indicated by their distance (nt) from the translations start sites of the pho84 and pho1 mRNAs. (B) To map the $5^{\prime}$ ends of the pho84 and prt RNAs, we analyzed the reverse transcriptase primer extension product (lanes *) in parallel with a series of DNA-directed primer extension reactions that contained mixtures of standard and chain-terminating nucleotides (the chain terminator is specified above the lanes). The primer extension products were analyzed by electrophoresis through a $42-\mathrm{cm}$ denaturing $8 \%$ polyacrylamide gel and visualized by autoradiography of the dried gel. The $5^{\prime}$ RNA ends are denoted by 4 to the right of the gel. The coding strand DNA sequences flanking the pho84 and prt transcription start sites are shown at right, with the start sites denoted by $户 .(C){ }^{32} \mathrm{P}$-labeled oligonucleotide primers complementary to phol and act1 mRNAs (left panel), pho84 mRNA RNA (top right panel), or prt RNA (bottom right panel) were annealed to total RNA from $p h o 7^{+}$(WT) or pho7s strains and extended with reverse transcriptase. The reaction products were analyzed by denaturing PAGE and visualized 
region between the phos4 and phol genes and extending through the pho1 open reading frame (Lee et al. 2013; Shah et al. 2014). This lncRNA, named prt, has been implicated as a negative regulator of pho1 expression (Lee et al. 2013; Shah et al. 2014). Here we report that the prt transcript is detectable in wild-type $r r p 6^{+}$cells by primer extension analysis with a ${ }^{32} \mathrm{P}$-labeled DNA primer complementary to the intergenic region. PAGE analysis of the reverse transcriptase primer extension product in parallel with a DNA-templated sequence ladder mapped the prt start site to an A position located 1198 nt upstream of the phol start codon (Fig. 3B). The $5^{\prime}$ end of prt in wild-type cells that we obtained by primer extension is at the same position reported for prt in $\operatorname{rrp} 6 \Delta$ cells, as determined by PCR amplification across the $5^{\prime} / 3^{\prime}$ junctions of decapped and intramolecularly ligated RNA circles, followed by cloning and sequencing of individual junctions (Shah et al. 2014).

\section{Differential requirements for Pho7 for expression of prt and the flanking mRNAs}

Primer extension analysis was used to gauge the role of Pho7 in the expression of the three transcription units that comprise the phosphate-responsive gene cluster. Total RNA from wild-type and pho7 $\Delta$ cells was annealed to a mixture of two ${ }^{32} \mathrm{P}$-labeled DNA primers complementary to mRNAs encoding actin and Phol. Discrete gene-specific extension products for both $\mathrm{mRNAs}$ were detected in a reaction programmed by RNA from wild-type cells, but only the actin product was detected in the reaction templated by RNA from pho7s cells (Fig. 3C). Primer extension reactions with a probe specific to pho84 revealed that transcription of the phosphate transporter gene was eliminated in pho7 $\Delta$ cells (Fig. 3C). In contrast, the prt primer extension product was unaffected by pho7 $\Delta$ (Fig. 3C). We conclude that prt transcription initiation is not dependent on the Pho7 transcription factor.

\section{Effects of Pol2 CTD mutations on expression of phosphate-responsive mRNAs}

The cell-based enzyme assays presented above showed that Phol was derepressed under phosphate-replete conditions by Pol2 CTD mutations that altered the number of Ser7 and Ser5 coding letters. To correlate these findings with transcription of the phosphate-responsive gene cluster, we analyzed RNA levels by primer extension templated by total RNA isolated from wild-type and mutant yeast strains that had been grown in liquid culture in YES medium, washed, and then incubated for $3 \mathrm{~h}$ either in synthetic medium containing $15.5 \mathrm{mM}$ phosphate (phosphate + ) or in medium lacking exogenous phosphate (phosphate -) (Fig. 4A). Actin mRNA was assayed as an internal control. We found that the phol mRNA and phos4 mRNA primer extension products were (i) increased under phosphate-replete condi- tions by the $S 7 A,(S 5)_{3}(S 5 A)_{11}$, and $(S 5)_{7}(S 5 A)_{7}$ CTD alleles that derepressed Phol enzymatic activity; and (ii) decreased under phosphate-replete conditions by the $T 4 A, T 4 A \cdot S 7 A$, and S7E CTD alleles that hyper-repressed basal acid phosphatase enzymatic activity (Fig. 4A). The phol and pho84 mRNAs were induced in wild-type, T4A, and T4A.S7A cells after $3 \mathrm{~h}$ of phosphate starvation, but the mRNA inductive response was blunted in S7E cells (Fig. 4A).

The radiolabeled phol primer extension product was quantified and normalized to the internal actin control, these values were then expressed as ratios to the level of pho1 mRNA in wild-type cells in phosphate-replete medium (Fig. 4B). We also performed independent measurements of phol mRNA levels by RT-qPCR with pho1-specific primers, again normalized to an internal actin mRNA control and expressed for each strain in $+/-$ phosphate conditions as the ratio to phol mRNA in wild-type cells in phosphaterich medium (Fig. 4C). The two methods revealed concordant mRNA expression patterns, whereby pho1 transcription is derepressed by CTD alleles $(S 5)_{3}(S 5 A)_{11}, S 7 A$, and $(S 5)_{7}(S 5 A)_{7}$ and hyper-repressed by $T 4 A, T 4 A \cdot S 7 A$, and $S 7 E$. The key point here is that the Ser7 and Ser5 CTD coding letters exert repressive effects on the Pho7-dependent mRNA transcription units under phosphate-replete conditions.

\section{Hyper-repression of Pho1 by CTD mutations correlates with elevated basal prt RNA level}

The prt RNA is implicated in repression of phol under phosphate-replete conditions, i.e., a deletion of the prt promoter element causes derepression of pho1 mRNA and phosphatase activity (Lee et al. 2013; Shah et al. 2014). We found here that the hyper-repressing rpb1-CTD-S7E allele increased the level of the prt RNA in phosphate-replete cells, as gauged by primer extension analysis (Fig. 4A), or by RT-qPCR, which indicated that the basal prt RNA level was 5.3-fold higher in S7E cells than in wild-type (Supplemental Fig. S2). The T4A and $T 4 A \cdot S 7 A$ alleles that reduced phol expression in phosphaterich medium also elicited 2.3-fold and 3.3-fold increases in prt RNA (Supplemental Fig. S2). The RT-qPCR data revealed lowered prt RNA levels $3 \mathrm{~h}$ after phosphate starvation of wildtype, T4A, and T4A•S7A cells (Supplemental Fig. S2), concomitant with the induction in phol mRNA levels at $3 \mathrm{~h}$ post-starvation (Fig. 4). Whereas the S7E mutant did evince a decrement in prt RNA at $3 \mathrm{~h}$ after phosphate starvation, the post-starvation level of prt was still 2.6-fold greater than that of wild-type cells in phosphate-replete medium (Supplemental Fig. S2), which might explain the attenuated induction of acid phosphatase activity (Fig. 1) and pho1 mRNA (Fig. 4) in phosphate-starved S7E cells. The derepressing S7A allele had little effect on prt RNA level in phosphate-replete cells (Supplemental Fig. S2), suggesting that the S7A effect is not solely via prt.

Previous microarray analysis of the transcriptional response of fission yeast as a function of time after transfer to 

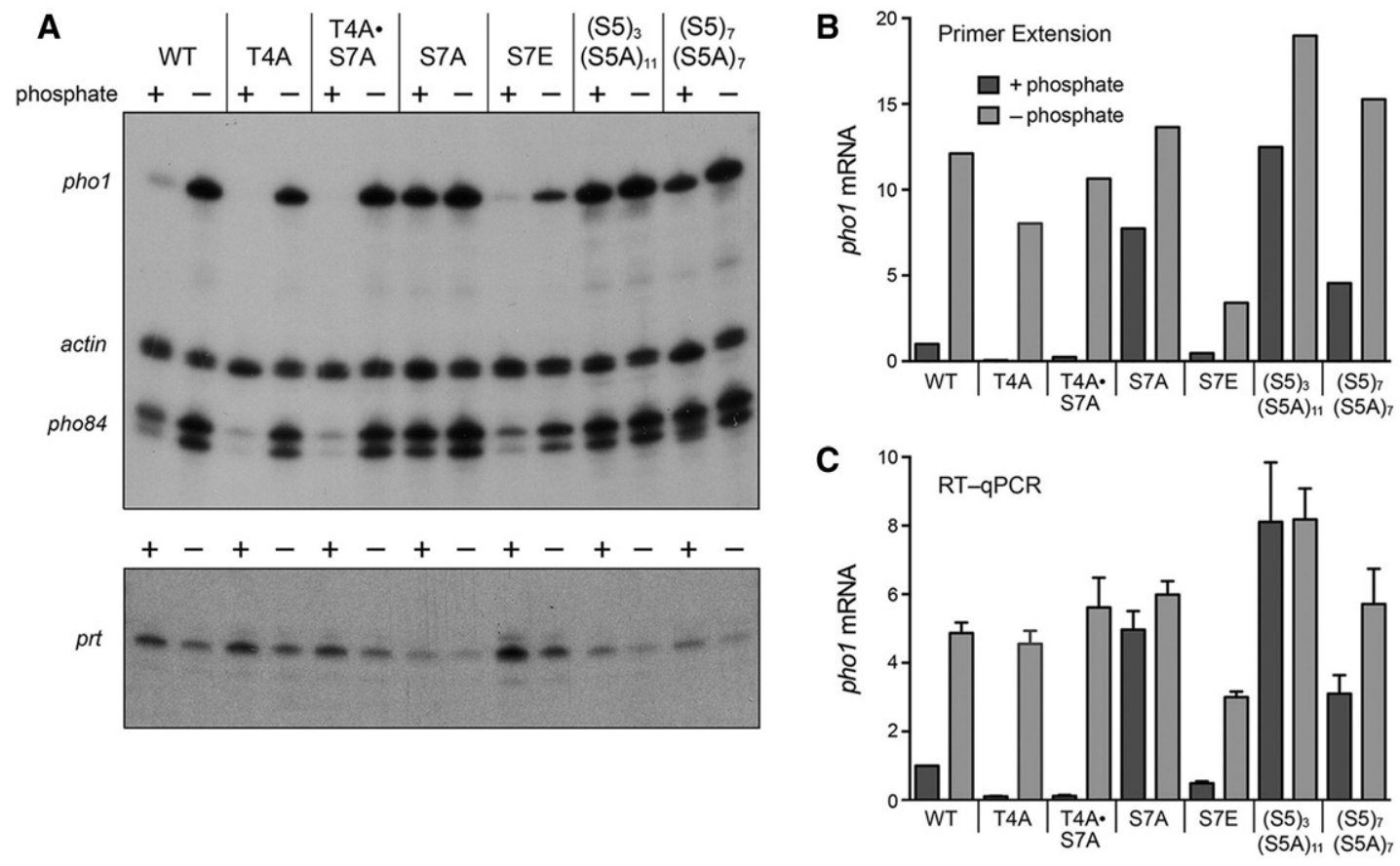

FIGURE 4. Effects of CTD mutations on expression of phosphate-responsive mRNAs. $(A){ }^{32}$ P-labeled primers complementary to pho1, pho84, and actl mRNAs (top panel) or prt RNA (bottom panel) were annealed to total RNA isolated from the indicated fission yeast strains that had been incubated for $3 \mathrm{~h}$ in PMG medium containing $15.5 \mathrm{mM}$ phosphate (+ phosphate) or lacking exogenous phosphate ( - phosphate). After primer extension with reverse transcriptase, the reaction products were analyzed by denaturing PAGE and visualized by autoradiography. (B) The pho1 primer extension product in panel A was quantified and normalized to that of act1 measured for the same RNA sample. The bar graph shows the fold-change in pho1 relative to the wild-type + phosphate control (defined as 1.0). (C) RT-qPCR analysis was performed as described in Materials and Methods. The level of pho1 transcript was normalized to that of act1 measured for the same RNA sample. The bar graph shows the fold-change in phol RNA relative to the wild-type + phosphate control (defined as 1.0). Each datum in the bar graph is the average of values from RT-qPCR analyses of RNAs from three independent yeast cultures. The error bars denote SEM.

phosphate-free medium (Carter-O'Connell et al. 2012) showed no increase in pho1 mRNAs after $30 \mathrm{~min}$, a twofold increase after $1 \mathrm{~h}$, and a fourfold induction after $2 \mathrm{~h}$. There is a lag between mRNA induction and the elaboration of active acid phosphatase on the surface of wild-type cells. Acid phosphatase was evident at $3 \mathrm{~h}$ after transfer to phosphate-free medium (but not at $2 \mathrm{~h}$ ) and then increased steadily up to at least $6 \mathrm{~h}$ (Supplemental Fig. S3), consistent with ongoing translation of the induced phol mRNA. These findings informed our choice of $3 \mathrm{~h}$ as a standard time to assess the effects of CTD mutations on pre- and post-starvation Phol expression. In the case of the T4A,T4A•S7A, and S7E mutants that reduced Phol activity at $3 \mathrm{~h}$ post-starvation, tracking the starvation response over a longer time frame $(6 \mathrm{~h})$ revealed that the induction of acid phosphatase activity was delayed, but not abolished (Supplemental Fig. S3). The T4A strain started to accrue acid phosphatase activity at 4 $\mathrm{h}$, which increased steadily thereafter, with a similar slope (albeit shifted to the right) as the wild-type strain (Supplemental Fig. S3). The T4A-S7A strain evinced a similar delay, with a slightly higher slope during recovery (conceivably because of the loss of the repressive Ser7 residue) (Supplemental Fig. S3). Because the T4A-mutated and wild-type cells had similar pho 1 mRNA levels at $3 \mathrm{~h}$ post-starvation, we surmise that the phosphatase activity lag reflects delayed onset of pho1 transcription induction by Pol2 lacking the Thr4 coding letter. The salient finding was that $S 7 E$ cells displayed a longer lag $(5 \mathrm{~h})$ and a more shallow increase in acid phosphatase activity (Supplemental Fig. S3), consistent with the lower pho1 mRNA level seen at $3 \mathrm{~h}$ after starvation in S7E cells.

\section{rrp6 $\Delta$ delays the induction of both pho1 and pho84}

Deletion of the gene encoding the Rrp6 subunit of the fission yeast nuclear exosome elicits the accumulation of prt IncRNA, as gauged by Northern blot analysis (Lee et al. 2013; Shah et al. 2014). Increased prt in turn dampens the induction of pho1 in response to phosphate starvation (Lee et al. 2013; Shah et al. 2014). In light of the present results showing the concordant effect of certain CTD mutations on phol and pho84 mRNA levels (Fig. 4A), we tracked by RT-qPCR and enzyme activity assays the phosphate starvation response in wild-type $r r p 6^{+}$and $\operatorname{rrp} 6 \Delta$ cells (Fig. 5). The onset of Phol phosphatase accumulation in $\operatorname{rrp} 6 \Delta$ cells occurred between 5 and $6 \mathrm{~h}$ after transfer to phosphate-free medium, i.e., with a $\sim 3-\mathrm{h}$ delay compared to the inflection point of Phol accumulation between 2 and $3 \mathrm{~h}$ post-transfer in the wild-type strain (Fig. 5A). Whereas pho1 and pho84 

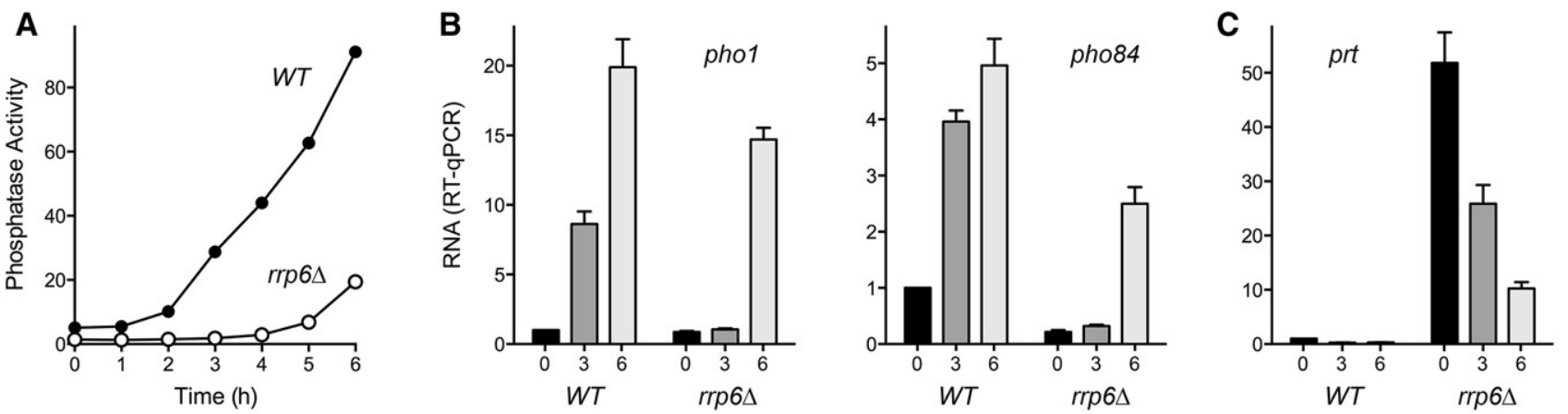

FIGURE 5. $r r p 6 \Delta$ delays the induction of both $p h o 1$ and $p h o 84$. (A) Fission yeast $r p b 1^{+} r r p 6^{+}(W T)$ and $r p b 1^{+} r r p 6 \Delta$ strains were grown in YES medium to $A_{600}$ of 0.5 to 0.7 . The cells were harvested, washed in water, and after withdrawing an aliquot to measure Phol activity (time 0 ), the cells were transferred to PMG medium lacking exogenous phosphate. Phol activity was assayed after incubation for the times specified. $(B, C)$ Total RNA isolated from $W T$ and $\operatorname{rrp} 6 \Delta$ cells at time 0 and at 3 and $6 \mathrm{~h}$ post-transfer to phosphate-free medium was used to assay by RT-qPCR the levels of pho1 and pho84 mRNAs $(B)$ and prt RNA $(C)$. The transcript levels were normalized to those of act1 measured for the same RNA samples. The bar graph shows the fold-change in RNA relative to the WT time 0 value (defined as 1.0). Each datum in the bar graphs is the average of values from RT-qPCR analyses of RNAs from three independent yeast cultures. The error bars denote SEM.

mRNA levels were induced in wild-type cells by $3 \mathrm{~h}$ post-starvation, there was no detectable increase in either transcript in rrp6 cells after $3 \mathrm{~h}$ of phosphate starvation (Fig. 5B). However, pho1 and pho84 mRNA levels did increase after $6 \mathrm{~h}$ poststarvation of $r r p 6 \Delta$ cells (Fig. 5B), signifying a concordantly delayed induction of both phosphate-responsive genes.

The level of the prt transcript was 50-fold higher in phosphate-replete $\operatorname{rrp} 6 \Delta$ cells versus wild-type, and declined by twofold and fivefold, respectively, after $3 \mathrm{~h}$ and $6 \mathrm{~h}$ of phosphate-starvation (Fig. 5C). The finding that $\operatorname{rrp} 6 \Delta$ cells managed to induce the phol and pho84 mRNAs at $6 \mathrm{~h}$ poststarvation, at which the prt RNA level was still 10-fold higher than in phosphate-replete wild-type cells, suggests that the mere presence of prt RNA transcribed from the prt locus in cis is not sufficient per se to keep the flanking phol and pho84 genes durably turned off when phosphate is limiting.

\section{Effects of manipulating protein kinases on Pho1 regulation}

Phol expression is strictly dependent on the yeast transcription factor Pho7 (Henry et al. 2011; Carter-O'Connell et al.

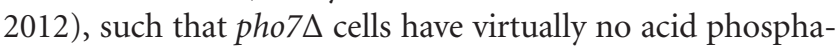
tase activity, even when starved for phosphate (Fig. 6). In contrast, csk $1 \Delta$ cells constitutively express acid phosphatase in phosphate-replete conditions (Henry et al. 2011; CarterO'Connell et al. 2012), at a level eightfold greater than wild type (Fig. 6). As noted previously (Henry et al. 2011; Carter-O'Connell et al. 2012), we find that all Phol expression in csk $1 \Delta$ cells is abolished by pho7 $($ Fig. 6), implying that the gain of Phol expression caused by failure of Csk1 to phosphorylate one or more of its physiological targets is effected through the normal Pho7-dependent transcription program, not via activation of a bypass pathway. Similarly, the derepression of Phol in rpb1-CTD-S7A and (S5A-S5) cells was abolished by pho7 (not shown).
The cyclin-dependent kinase Cdk9 is a physiological target for activation by Csk1 (Pei et al. 2006). Cdk9, in a complex with its cyclin partner Pch1, catalyzes serine phosphorylation of the Pol2 CTD (Pei and Shuman 2003; Pei et al. 2003). Csk1 stimulates the CTD kinase activity of Cdk $9 \cdot P c h 1$ by phosphorylating Cdk9 on residue Thr212 of the activating "Tloop" segment. The activated Cdk9•Pch1 enzyme phosphorylates the Pol2 CTD at positions Ser2 and Ser5 of the CTD heptad (Pei et al. 2006). The Cdk9-T212A mutant is refractory to activation by Csk 1 . Indeed, fission yeast $c s k 1 \Delta$ and $c d k 9$ T212A strains phenocopy each other with respect to their poor growth on minimal media and cold-sensitivity on rich medium (Pei et al. 2006). The key question here is: Do the csk $1 \Delta$ and $c d k 9-T 212 A$ mutations elicit similar effects on phosphate homeostasis?

Pho1 was constitutively expressed in $c d k 9-T 212 \mathrm{~A}$ cells (sevenfold greater than wild-type) (Fig. 6). Moreover, the derepression of Pho1 by $c d k 9-T 212 A$ was effaced by pho7 $\Delta$

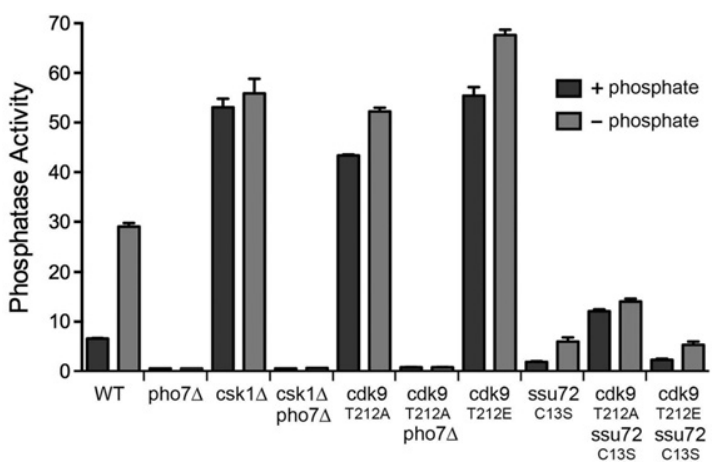

FIGURE 6. Effects of manipulating Csk1 and Cdk9 kinases and Ssu72 phosphatase on Phol regulation. $r p b 1^{+}$cells, either wild-type or bearing additional mutations as specified, were assayed for Phol activity after incubation for $3 \mathrm{~h}$ in PMG medium containing $15.5 \mathrm{mM}$ phosphate (+ phosphate) or lacking exogenous phosphate (- phosphate). 
(Fig. 6). These results suggest that Csk1 acts through Cdk9 to repress Phol expression under phosphate-replete conditions.

The phosphomimetic Cdk9 mutation T212E enhances the kinase activity of recombinant Cdk9・Pch1 about threefold compared with the wild-type enzyme that had not been activated by Csk1 and to the T212A mutant enzyme. Nonetheless, the kinase activity of Cdk9-T212E・Pch1 is threefold lower than that of wild-type Cdk9-Pch1 that had been activated by Csk1 (Pei et al. 2006). Thus Cdk9-T212E appears to be a hypomorphic mutation (Pei et al. 2003). In that vein, it is noteworthy that Phol was expressed constitutively in $c d k 9-T 212 E$ cells (Fig. 6).

\section{Phosphatase Ssu72 is important for Pho1 expression}

The CTD phosphatase Ssu72 is an agent of Ser5 and Ser7 dephosphorylation in the budding yeast Saccharomyces cerevisiae (Krishnamurthy et al. 2004; Hausmann et al. 2005; Bataille et al. 2012; Zhang et al. 2012). Ssu72 catalyzes phosphoryl transfer via a covalent enzyme-cysteinyl-S-phosphate intermediate; mutation of the active site cysteine to serine or alanine abolishes phosphatase activity. The Ssu72 protein and its phosphatase activity are essential for the viability of $S$. cerevisiae (Krishnamurthy et al. 2004). In contrast, S. pombe Ssu72 (SPAC3G9.04) is dispensable for growth of fission yeast (Schwer et al. 2012). To gauge the potential contribution of Ssu72 to fission yeast phosphate homeostasis, we replaced the wild-type ssu $72^{+}$gene with ssu72-C13S, in which the cysteine nucleophile was mutated to serine. We found that ssu72-C13S cells had diminished basal Pho1 activity and a feeble inductive response to phosphate starvation compared with wild-type cells (Fig. 6; Supplemental Fig. S3). RNA analysis by primer extension revealed that the ssu72C13S mutation elicited hyper-repression of the phol and pho84 mRNAs under phosphate-replete conditions and attenuated the transcriptional response to phosphate depletion, such that the levels of pho1 and pho84 mRNAs achieved in C13S cells at $3 \mathrm{~h}$ post-starvation were about the same as those in nonstarved wild-type cells (Supplemental Fig. S4).
Moreover, ssu72-C13S eliminated the high levels of Phol seen in $c d k 9$ mutants T212A and T212E (Fig. 6). We infer that Ssu72 phosphatase activity is important for Phol expression, even when a repressive arm of the regulatory circuit is removed.

\section{Genetic interaction of Ssu72 with the Pol2 CTD}

In an effort to connect the Ssu72 effect on Pho1 expression with CTD phosphorylation status, we performed Western blotting of total protein from ssu $72^{+}$and ssu72-C13S cells with CTD phospho-specific antibodies. However, this analysis revealed no changes in the bulk phosphorylation signals for the Ser5- $\mathrm{PO}_{4}$ or Ser7- $\mathrm{PO}_{4}$ marks (not shown). To query a genetic interaction between Ssu72 and the Pol2 CTD, we tested for mutational synergy between ssu72-C13S and our collection of $r p b 1$ alleles with serially truncated CTDs. We found that shortening the CTD to 20, 18 and 16 repeats in the ssu72-C13S strain elicited progressively worsening coldsensitive and temperature-sensitive growth defects (Fig. $7 \mathrm{~A}$ ). Indeed, a reduction to 16 repeats (rump plus 12 consensus heptads) rendered the $s s u 72-C 13 S$ strain inviable at $18^{\circ} \mathrm{C}$, $20^{\circ} \mathrm{C}$, and $37^{\circ} \mathrm{C}$ and barely viable at $30^{\circ} \mathrm{C}$ and $34^{\circ} \mathrm{C}$. The salient finding was that the cs and ts defects of ssu72-C13S in the context of CTD shortened to 18 repeats (rump plus 14 consensus heptads) were suppressed by mutating the 14 Ser7 positions to alanine, i.e., $S 7 A$ restored wild-type growth at $30^{\circ} \mathrm{C}, 34^{\circ} \mathrm{C}$, and $37^{\circ} \mathrm{C}$ to ssu $72-\mathrm{C} 13 \mathrm{~S}$ cells with 18 repeats (as gauged by colony size) and enhanced growth at $18^{\circ} \mathrm{C}$, $20^{\circ} \mathrm{C}$, and $25^{\circ} \mathrm{C}$ (Fig. 7A). An identical synthetic growth defect ( $c s$ and $t s$ ) was observed when the CTD was shortened to 18 repeats in the $s s u 72 \Delta$ null strain, which was suppressed in the identical fashion by $S 7 A$ (not shown). These results establish that the phosphatase activity of Ssu72 becomes critical for fission yeast growth when CTD length is shortened. Relaxation of this requirement by $S 7 A$ (which precludes deposition of the Ser7- $\mathrm{PO}_{4}$ mark) raises the prospect that Ssu72 might be an agent of Ser7 dephosphorylation in vivo in fission yeast.
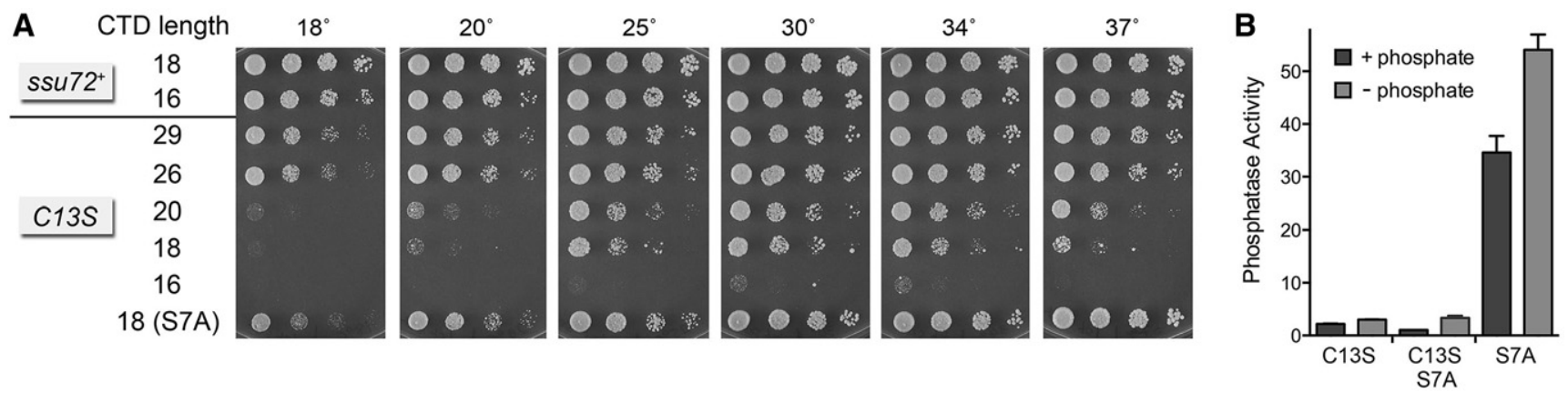

FIGURE 7. Genetic and functional interaction of Ssu72 with the Pol2 CTD. (A) Exponentially growing cultures of $S$. pombe strains with the indicated chromosomal $r p b 1-C T D$ and ssu 72 alleles were adjusted to $A_{600}$ of 0.1 , and aliquots of serial fivefold dilutions were spotted to YES agar and incubated at the indicated temperatures. (B) Fission yeast cells with genotypes as specified were assayed for Phol activity after incubation for 3 h in PMG medium containing $15.5 \mathrm{mM}$ phosphate (+ phosphate) or lacking exogenous phosphate ( - phosphate). 
When we tested the ssu72-C13S rpb1-CTD-S7A strain for Phol expression, we found that inactivation of the Ssu72 enzyme effaced the constitutive expression of Phol that was characteristic of the $S 7 A$ strain (Fig. 7B). ssu72-C13S rpb1-CTD-S7A cells resembled the ssu72-C13S strain with respect to decreased basal Phol activity and feeble induction by phosphate starvation (Fig. 7B). This result signifies that the requirement for Ssu72 for Pho1 expression cannot simply be for dephosphorylation of Ser7 (i.e., if that were the case, then ssu72-C13S rpb1-CTD-S7A ought to have phenocopied $S 7 A$ ).

\section{DISCUSSION}

The present study sheds new light on the fission yeast CTD code in general and its particular role in phosphate homeostasis. The information content of the code is multilayered. The most basic components are the 12 individual "letters" of the consensus heptad: Tyr1/Tyr1- $\mathrm{PO}_{4}$, Ser2/Ser2- $\mathrm{PO}_{4}$, Pro3, Thr4/Thr4-PO, Ser5/Ser5- $\mathrm{PO}_{4}$, Pro6, and Ser7/Ser7$\mathrm{PO}_{4}$. Systematic CTD mutagenesis by alanine scanning and conservative substitutions has established that seven of the letters, including the Tyr1- $\mathrm{PO}_{4}, \mathrm{Ser} 2-\mathrm{PO}_{4}, \mathrm{Thr} 4-\mathrm{PO}_{4}$, and Ser7- $\mathrm{PO}_{4}$ marks, are inessential for fission yeast vegetative growth (Schwer and Shuman 2011; Schwer et al. 2012). Interrogating several of the rpb1-CTD mutants by RNA-seq revealed how individual coding letters govern distinct gene expression programs (Schwer et al. 2014). Insights to the vocabulary of the code emerged when mutations in two coding letters coordinately affected the expression of a common set of genes, in which case the two letters might comprise a coding "word" read by proteins that control expression of the shared target gene set. This was the case for the $Y 1 F$ and $S 2 A$ mutations. The most striking expression signature of the $Y 1 F$ and $S 2 A$ alleles was the derepression of the multigene iron homeostasis regulon (Schwer et al. 2014), which is normally silenced under iron-replete conditions by the DNA-binding transcription factor Fep1 (Labbé et al. 2013). We proposed that the two-letter CTD Tyr1-Ser2 coding word is necessary to transduce a repressive signal from Fep 1 to the transcription apparatus.

Here we expanded on earlier findings that implicated the Pol2 CTD in the expression of phosphate-regulated genes. The salient new data implicate Ser5, Pro6, and Ser7 as component letters of a CTD word needed to dampen Phol expression under phosphate-replete conditions. The opposite effects of the $S 7 A$ mutation that erases the Ser7- $\mathrm{PO}_{4}$ mark (derepression of Phol in the presence of phosphate) versus the S7E mutation that mimics the Ser7- $\mathrm{PO}_{4}$ mark (hyper-repression of Phol when phosphate is available and feeble induction of Phol during phosphate starvation) suggest that Ser7- $\mathrm{PO}_{4}$ elicits Phol silencing.

Assigning a gene expression signature to the Ser5 and Ser5$\mathrm{PO}_{4}$ letters is complicated by the fact that replacing all Ser5 positions with alanine is lethal, because Ser5- $\mathrm{PO}_{4}$ is essential to recruit the mRNA capping apparatus to the Pol2 elongation complex. Here we exploited truncated CTDs and chimeric S5•S5A CTDs with progressively reduced Ser5 content to provide the first clues to the contributions of Ser5 to a specific regulatory circuit. Whereas truncating the CTD to contain a rump plus nine consensus Ser5-containing heptads had no impact on regulated Phol expression (Supplemental Fig. S1, the $n=13$ strain), a chimeric $(\mathrm{S} 5)_{n}(\mathrm{~S} 5 \mathrm{~A})_{14-n}$ CTD containing seven or fewer Ser5 heptads resulted in derepression of Phol in phosphate-rich medium and hyper-induction during starvation. The degree of derepression increased steadily as " $n$ " was decreased from seven to two Ser5 heptads.

As we show here, reduced Ser 5 content mimicked S7A with respect to dysregulation of Phol expression. We speculate that Ser5 content and phosphorylation status might exert its effect on phosphate homeostasis, in some part, via Ser7 and its phosphorylation, insofar as (i) mutating all Ser5 to alanine effaces the Ser7- $\mathrm{PO}_{4}$ mark; (ii) mutating all Ser7 to alanine has no discernible effect on the Ser5- $\mathrm{PO}_{4}$ mark; and (iii) the signal intensity of the Ser5- $\mathrm{PO}_{4}$ and Ser7- $\mathrm{PO}_{4}$ marks both increase in proportion to Ser 5 heptad content in the chimeric (S5) $(\mathrm{S} 5 \mathrm{~A})_{14-\mathrm{n}}$ strains (Schwer et al. 2012). Similarly, we infer that $P 6 A$ dysregulates Phol expression in the same fashion as $S 5 A$ and $S 7 A$ because $P 6 A$ precludes inscription of the Ser5- $\mathrm{PO}_{4}$ mark (Schwer et al. 2012).

The prominent role of CTD phosphorylation in silencing Phol under replete conditions resonates with the findings that mutations of Csk1 and Cdk9, which together comprise a two-kinase cascade that phosphorylates the CTD, also derepress Phol expression in replete medium. In contrast, inactivation of the CTD phosphatase Ssu72 blocks Pho1 expression, and does so even in genetic backgrounds that elicit constitutive Pho1 induction (e.g., rpb1-CTD-S7A and $c d k 9$ $T 212 E$ ). Because the biochemical activity and substrate specificity of fission yeast Ssu72 are uncharted (and because our efforts to produce recombinant S. pombe Ssu72 in fission yeast in E. coli have yielded intractably insoluble protein), we cannot speculate presently as to the identity of the phosphoprotein substrate(s) on which Ssu72 must act to promote Phol expression.

Finally, our results here confirm and add to recent studies of Lee et al. (2013) and/or Shah et al. (2014) regarding the negative influence of prt lncRNA on the phosphate starvation response. We find that (i) prt is detectable by primer extension and RT-qPCR assays in wild-type fission yeast under phosphate-replete conditions; (ii) prt level is 50-fold higher in $\operatorname{rrp} 6 \Delta$ cells; (iii) the $5^{\prime}$ ends of prt are the same in $r r p 6^{+}$ and $\operatorname{rrp} 6 \Delta$ cells; (iv) the high prt level that accumulates in $\operatorname{rrp} 6 \Delta$ cells causes a kinetic delay in the pho1 inductive response (by $\sim 3 \mathrm{~h}$ ) but does not durably prevent the induction; and (v) the hyper-repression of Phol by CTD mutations $S 7 E, T 4 A$, and $T 4 A \cdot S 7 A$ in the presence of phosphate correlates with increased levels of prt RNA. Here we note that the $\operatorname{rrp} 6 \Delta$ mutation elicits similar effects on the upstream pho84 gene of the phosphate-responsive locus, 
manifest by hyper-repression of pho84 in phosphate-replete rrp6 6 cells and delayed induction of pho84 mRNA after phosphate starvation. This result raises the prospect that the prt lncRNA acts as a repressor of both upstream and downstream protein-coding genes. (Alternatively, there could be another regulatory RNA, distinct from prt but also increased in $r r p 6 \Delta$ cells, that controls pho84 expression.) Although the mechanism of prt action is not fully understood, current models posit that cis-acting elements in the nascent prt transcript recruit the exosome and RNAi machinery, which in turn establish a repressive state at the pho1 gene (Lee et al. 2013; Shah et al. 2014). Our results implicate Pol2 itself (via the CTD) as a key player in controlling the phosphate starvation response.

\section{MATERIALS AND METHODS}

\section{Assay of induction of acid phosphatase activity after phosphate starvation}

Aliquots of exponentially growing S. pombe cultures in YES (yeast extract with supplements) medium were harvested, the cells were washed in water and adjusted to $A_{600}$ of $\sim 0.3$ in PMG (Pombe glutamate) medium, either with $15.5 \mathrm{mM}$ phosphate $(+)$ or without phosphate (-). After incubation for $3 \mathrm{~h}$ at $30^{\circ} \mathrm{C}$, cells were harvested, washed and suspended in water to attain $A_{600}$ of 1.25 . To quantify acid phosphatase activity, reaction mixtures $(200 \mu \mathrm{L})$ containing $100 \mathrm{mM}$ sodium acetate ( $\mathrm{pH} 4.2), 10 \mathrm{mM}$ p-nitrophenylphosphate, and serial twofold dilutions of cells (ranging from 0.00625 to 0.1 $A_{600}$ units) were incubated for $5 \mathrm{~min}$ at $30^{\circ} \mathrm{C}$. The reactions were quenched by adding $1 \mathrm{~mL}$ of $1 \mathrm{M}$ sodium carbonate, the cells were removed by centrifugation, and the absorbance of the supernatant at $410 \mathrm{~nm}$ was measured. Acid phosphatase activity is expressed as the ratio of $A_{410}$ ( $p$-nitrophenol production) to $A_{600}$ (cells). Each datum in the bar graphs is the average of three assays using cells from three independent cultures \pm SEM.

\section{RNA analyses}

Total RNA was extracted via the hot phenol method from $15 A_{600}$ units of yeast cells that had been incubated for $3 \mathrm{~h}$ at $30^{\circ} \mathrm{C}$ in PMG medium with $15.5 \mathrm{mM}$ phosphate $(+)$ or without phosphate (-). For RT-qPCR, the RNAs were treated with DNase I, extracted serially with phenol:chloroform and chloroform, and then precipitated with ethanol. The RNAs were resuspended in $10 \mathrm{mM}$ Tris- $\mathrm{HCl}$ (pH 6.8), 1 mM EDTA and adjusted to a concentration $600 \mathrm{ng} / \mu \mathrm{L}$. Reverse transcription (RT) and gene-specific quantitative PCR (qPCR) were performed as previously described (Schwer et al. 2014). Primer extension assays (Schwer et al. 2014) were programmed by $10 \mu \mathrm{g}$ (for analysis of pho1, pho84, and act1) or $20 \mu \mathrm{g}$ (for prt) of total yeast RNA as templates for M-MuLV reverse transcriptase-catalyzed elongation of $5^{\prime}{ }^{32} \mathrm{P}$-labeled oligodeoxynucleotide primers complementary to the transcripts of interest (see Supplemental Table S1 for primer sequences).

\section{SUPPLEMENTAL MATERIAL}

Supplemental material is available for this article.

\section{ACKNOWLEDGMENTS}

This research was supported by National Institutes of Health grant GM52470.

Received May 15, 2015; accepted July 8, 2015.

\section{REFERENCES}

Bataille AR, Jeronimo C, Jacques PÉ, Laramée L, Fortin ME, Forest A, Bergeron M, Hanes SD, Robert F. 2012. A universal RNA polymerase II CTD cycle is orchestrated by complex interplays between kinase, phosphatase, and isomerase enzymes along genes. Mol Cell 45: $158-170$.

Carter-O'Connell I, Peel MT, Wykoff DD, O’Shea EK. 2012. Genomewide characterization of the phosphate starvation response in Schizosaccharomyces pombe. BMC Genomics 13: 697.

Corden JL. 2013. RNA polymerase II C-terminal domain: tethering transcription to transcript and template. Chem Rev 113: 84238455.

Doamekpor SK, Sanchez AM, Schwer B, Shuman S, Lima CD. 2014. How an mRNA capping enzyme reads distinct RNA polymerase II and Spt5 CTD phosphorylation codes. Genes Dev 28: $1323-1336$.

Eick D, Geyer M. 2013. The RNA polymerase II carboxy-terminal domain (CTD) code. Chem Rev 113: 8456-8490.

Hausmann S, Koiwa H, Krishnamurthy S, Hampsey M, Shuman S. 2005. Different strategies for carboxyl-terminal domain (CTD) recognition by Serine5-specific CTD phosphatases. J Biol Chem 280: 37681-37688.

Henry TC, Power JE, Kerwin CL, Mohammed A, Weissman JS, Cameron DM, Wykoff DD. 2011. Systematic screen of Schizosaccharomyces pombe deletion collection uncovers parallel evolution of the phosphate signal pathways in yeasts. Eukaryot Cell 10: 198-206.

Krishnamurthy S, He X, Reyes-Reyes M, Moore C, Hampsey M. 2004. Ssu72 is an RNA polymerase II CTD phosphatase. Mol Cell 14: 387-394.

Labbé S, Khan GM, Jacques JF. 2013. Iron uptake and regulation in Schizosaccharomyces pombe. Curr Opin Microbiol 16: 669-676.

Lee NN, Chalamcharia VR, Reyes-Turce F, Mehta S, Zofall M, Balachandran V, Dhakshnamoorthy J, Taneja N, Yamanaka S, Zhou M, et al. 2013. Mtr4-like protein coordinates nuclear RNA processing for heterochromatin assembly and for telomere maintenance. Cell 155: 1061-1074.

MacPherson S, Larochelle M, Tyrcotte B. 2006. A fungal family of transcriptional regulators: the zinc cluster proteins. Microbiol Mol Biol Rev 70: 583-604.

Pei Y, Shuman S. 2003. Characterization of the Schizosaccharomyces pombe Cdk9/Pch1 protein kinase: Spt5 phosphorylation, autophosphorylation, and mutational analysis. J Biol Chem 278: 43346-43356.

Pei Y, Schwer B, Shuman S. 2003. Interactions between fission yeast Cdk9, its cyclin partner Pch1, and mRNA capping enzyme Pctl suggest an elongation checkpoint for mRNA quality control. J Biol Chem 278: 7180-7188.

Pei Y, Du H, Singer J, St Amour C, Granitto S, Shuman S, Fisher RP. 2006. Cyclin-dependent kinase 9 (Cdk9) of fission yeast is activated by the CDK-activating kinase Csk1, overlaps functionally with the TFIIH-associated kinase Mcs6, and associates with the mRNA cap methyltransferase Pcm1 in vivo. Mol Cell Biol 26: 777-788.

Saiz JE, Fisher RP. 2002. A CDK-activating kinase network is required in cell cycle control and transcription in fission yeast. Curr Biol 12: $1100-1105$.

Schneider S, Pei Y, Shuman S, Schwer B. 2010. Separable functions of the fission yeast Spt5 carboxyl-terminal domain (CTD) in capping enzyme binding and transcription elongation overlap with those of the RNA polymerase II CTD. Mol Cell Biol 30: 2353-2364. 


\section{Schwer et al.}

Schwer B, Shuman S. 2011. Deciphering the RNA polymerase II CTD code in fission yeast. Mol Cell 43: 311-318.

Schwer B, Sanchez AM, Shuman S. 2012. Punctuation and syntax of the RNA polymerase II CTD code in fission yeast. Proc Natl Acad Sci 109: 18024-18029.

Schwer B, Bitton DA, Sanchez AM, Bähler J, Shuman S. 2014. Individual letters of the RNA polymerase II CTD code govern distinct gene expression programs in fission yeast. Proc Natl Acad Sci 111: 4185-4190.

Shah S, Wittmann S, Kilchert C, Vasiljeva L. 2014. IncRNA recruits RNAi and the exosome to dynamically regulate phol expression in response to phosphate levels in fission yeast. Genes Dev 28: 231-244

Viladevall L, St Amour CV, Rosebrock A, Schneider S, Zhang C, Allen JJ, Shokat KM, Schwer B, Leatherwood JK, Fisher RP. 2009. TFIIH and $\mathrm{P}-\mathrm{TEFb}$ coordinate transcription with capping enzyme recruitment at specific genes in fission yeast. Mol Cell 33: 738-751.

Zhang DW, Mosley AL, Ramisetty SR, Rodríguez-Molina JB, Washburn MP, Ansari AZ. 2012. Ssu72 phosphatase-dependent erasure of phospho-Ser7 marks on the RNA polymerase II C-terminal domain is essential for viability and transcription termination. J Biol Chem 287: 8541-8551. 

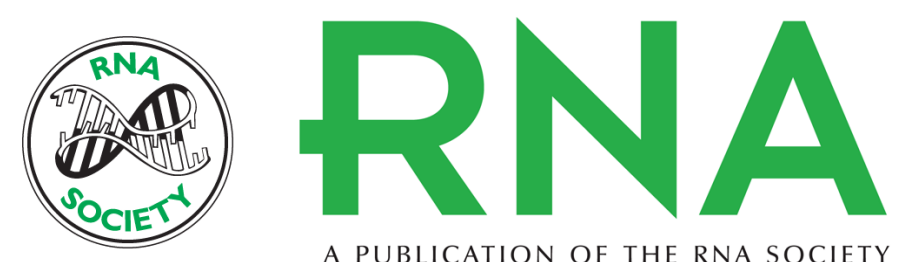

A PUBLICATION OF THE RNA SOCIETY

\section{RNA polymerase II CTD phospho-sites Ser5 and Ser7 govern phosphate homeostasis in fission yeast}

Beate Schwer, Ana M. Sanchez and Stewart Shuman

RNA 2015 21: 1770-1780 originally published online August 11, 2015

Access the most recent version at doi:10.1261/rna.052555.115

\section{Supplemental http://rnajournal.cshlp.org/content/suppl/2015/07/29/rna.052555.115.DC1 Material}

References This article cites 22 articles, 12 of which can be accessed free at: http://rnajournal.cshlp.org/content/21/10/1770.full.html\#ref-list-1

Creative This article is distributed exclusively by the RNA Society for the first 12 months after the Commons License full-issue publication date (see http://rnajournal.cshlp.org/site/misc/terms.xhtml). After 12 months, it is available under a Creative Commons License (Attribution-NonCommercial 4.0 International), as described at http://creativecommons.org/licenses/by-nc/4.0/.
Email Alerting Receive free email alerts when new articles cite this article - sign up in the box at the Service top right corner of the article or click here.

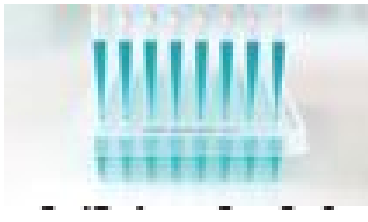

\section{Providing Precise Solutions for} your research.

To subscribe to $R N A$ go to:

http://rnajournal.cshlp.org/subscriptions 